\title{
Osteopatia hipertrófica secundária a osteossarcoma condroblástico extraesquelético em um cão
}

\author{
Hypertrophic Osteopathy Secondary to Extraskeletal Chondroblastic Osteosarcoma in a Dog \\ Natalia Martins Hoffmann', Mayron Tobias da Luz², Luisa LemosVieira ${ }^{3}$, Matheus Folgearini Silveira', \\ Pâmela da Silva Corrêa ${ }^{3} \&$ Débora Maria Marques Callado de Oliveira ${ }^{2}$
}

\begin{abstract}
Background: hypertrophic osteopathy is a periosteum disturb characterized by diffuse new bone formation which leads to significant thickening and deformity of members. Secondary in nature, it usually follows large pulmonary lesions such as abscesses and neoplasms. Extraskeletal osteosarcomas are rare and extremely malignant mesenchymal neoplasms. They comprise approximately $1 \%$ of all domestic animals' osteosarcomas and develop in the absence of a primary bone lesion. The aim of this paper was to describe a case of hypertrophic osteopathy, involving joints and upper limbs bones including ilium, secondary to a mediastinal chondroblastic osteosarcoma with pulmonary metastasis.

Case: A 10-year-old spayed female mixed breed dog, weighing $9 \mathrm{~kg}$, was presented with painful limbs, lameness, hind limbs swelling and a four-month history of weight loss. Radiographic examination revealed bilateral and asymmetric periosteal reactions on diaphyseal and/or epiphyseal areas of all proximal phalanges; metacarpal, metatarsal, carpal and tarsal bones; radius; ulna; tibia; fibula; humerus; femur and right ilium. An increased soft tissue radiopacity was noted on the lateral side of the right knee joint. Thoracic radiographies and ultrasonography suggested the presence of a 5-cm neoplasm or abscess in the left caudal lung lobe. At necropsy, the lobe showed a firm and solid, oval white mass measuring $5.2 \times 2.9 \mathrm{~cm}$. Another mass was found in the caudal mediastinum, near the diaphragm, with same color and more irregular aspect, measuring 3.3 $\mathrm{cm}$ of diameter. Intense periosteal new-bone formation was seen in the entire length of the four limbs bones, characterized by thickening of the bone surface and formation of irregular trabeculae perpendicular to the cortex. Significant swelling and thickening of the joint capsule was noted in the right knee. There was no microbial growth on aerobic or anaerobic cultures from the masses samples sent to culture. Histopathological examination showed areas of chondroid differentiation, osteoid tissue formation and cell morphology suggestive of chondroblastic osteosarcoma in mediastinal region, with invasion and involvement of the diaphragm and lungs. The analyzed bone fragment had large foci of tissue compaction, peritrabecular bleeding and mineralization of osteoid tissue, permeated by plasma cells and typical lymphocytes.

Discussion: Although hypertrophic osteopathy is often characterized as a disease which affects the diaphysis of distal long bones, this case presented a proximal progression of the disease. There was an unusual involvement of joints and ilium, which reinforces the importance of radiographic evaluation of these regions. Further studies on the pathogenesis of the syndrome are required, as its exact mechanisms remain obscure. It is suggested that the term hypertrophic osteoarthropathy should not be consider a misnomer since joint involvement is not exclusive of human form of the disease. Mediastinal masses are important cause of hypertrophic osteopathy. However, this is the first paper the authors are aware of that reports the occurrence of hypertrophic osteopathy secondary to mediastinal osteosarcoma. Finally, although rare, extra skeletal osteosarcoma should be considered in the differential diagnosis of intrathoracic masses in dogs with hypertrophic osteopathy. Timely diagnosis of hypertrophic osteopathy, whose signs of lameness and painful limbs draw the owner's attention, may favor the diagnosis of severe concomitant diseases.
\end{abstract}

Keywords: hypertrophic pulmonary osteoarthropathy, mediastinal osteosarcoma, paraneoplastic syndrome, canine. Descritores: osteoartropatia pulmonar hipertrófica, osteossarcoma mediastínico, síndrome paraneoplásica, canino. 


\section{INTRODUÇÃO}

Osteossarcomas extraesqueléticos são tumores mesenquimais malignos produtores de matriz osteoide, sem envolvimento periosteal ou ósseo primário [5,8]. A ocorrência de osteossarcoma extraesquelético é rara, representando aproximadamente $1 \%$ dos casos de osteossarcomas em animais domésticos [14]. Em cães, é relatado em diferentes órgãos e tecidos, incluindo fígado, baço, rim, mesentério, omento e glândula mamária [5].

A osteopatia hipertrófica $(\mathrm{OH})$, também conhecida como doença de Marie, osteopatia hipertrófica pulmonar ou osteoartropatia hipertrófica, é uma síndrome secundária à presença de uma massa em tórax ou abdômen, ou à doença cardiovascular, caracterizada por neoformação óssea crônica, que produz significativo espessamento e deformidade nos membros $[2,3,17]$. Causas não neoplásicas, incluem doenças pulmonares, corpos estranhos intratorácicos, dirofilariose ,espirocercose e tuberculose [1]. A OH é uma doença de ocorrência incomum, de maior incidência em cães e em humanos, sendo também descrita em outras espécies [2]. Sua patogenia, ainda não completamente esclarecida, é relacionada à diferente mecanismos, que possuem como fator comum o aumento do fluxo sanguíneo distal nos membros [3].

Alguns autores ainda definem a $\mathrm{OH}$ como doença que acomete as diáfises de ossos longos distais dos membros. Entretanto, o acometimento de outras regiões anatômicas é descrito neste relato. Objetivouse descrever um caso de osteopatia hipertrófica com envolvimento de articulações e ossos proximais do esqueleto apendicular, secundário a osteossarcoma condroblástico mediastínico em cão, correlacionando os achados clínicos, radiográficos e anatomopatológicos.

\section{CASO}

Um canino fêmea, esterilizado, sem raça definida, de 10 anos de idade e pesando $9 \mathrm{~kg}$, foi atendido no Hospital Veterinário da Universidade do Sul de Santa Catarina (HV-UNISUL) com histórico de dificuldade de deambulação, dor e aumento de volume em membros pélvicos, e perda de peso há quatro meses. $\mathrm{O}$ exame radiográfico revelou aumento de radiopacidade de tecidos moles lateral à articulação do joelho direito. Reações periosteais bilaterais e assimétricas do tipo lamelar ou paliçada foram observadas em regiões diafisárias e também epifisárias de úmero, rádio, ulna, fêmur, tíbia, fíbula, patela, ossos do carpo e tarso, metacarpos, metatarsos e falanges (Figura 1A). Neoformação periosteal similar foi verificada ao longo de todo o ílio direito. Radiografias torácicas e ultrassonografia utilizando as janelas acústicas do $6^{\circ}$ ao $8^{\circ}$ espaço intercostal esquerdo, sugeriram presença de neoplasia ou abscesso localizado no lobo pulmonar caudal esquerdo de aproximadamente $5 \mathrm{~cm}$. Com os achados da clínica, associado aos resultados radiográficos, sugeriu-se o diagnóstico de osteopatia hipertrófica. Foram prescritos analgésicos cloridrato de tramadol (Cronidor $\left.{ }^{1}\right)^{1}$ [ $5 \mathrm{mg} / \mathrm{kg}$, a cada $8 \mathrm{~h}$ ], dipirona (Dipirona Gotas Biovet ()$^{2}{ }^{2}[25 \mathrm{mg} / \mathrm{kg}$, a cada $8 \mathrm{~h}]$ e antiinflamatório esteroidal prednisona (Meticorten $\left.{ }^{\circledR}\right)^{3}[1 \mathrm{mg} / \mathrm{kg}$, a cada 24 h], por 5 dias, e indicada a lobectomia pulmonar caudal esquerda. Dois dias após a consulta, o paciente foi atendido em uma clínica veterinária em sua cidade de origem apresentando grave dispneia, sialorréia e permanência em decúbito lateral. Optou-se então pela eutanásia.

À necropsia, neoformações ósseas periosteais caracterizadas por espessamento da superfície óssea e formação de trabéculas irregulares, perpendiculares ao córtex, foram visibilizadas em toda a extensão dos ossos dos membros. As alterações foram mais evidentes após a maceração dos ossos [3], e remoção do espesso periósteo, que revelou aspecto de couveflor em diversas superfícies ósseas (Figura 1 B-D). O comprometimento articular ficou evidente pela hiperostose patelar e em regiões de epífise próximas a superfícies articulares, bem como pelo aumento de volume em joelho direito, com grande espessamento da cápsula articular. No mediastino caudal, junto à inserção do diafragma, constatou-se uma massa de coloração branca e consistência firme, com $3,3 \mathrm{~cm}$ de diâmetro e superfície irregular (Figura 2A). O lobo caudal esquerdo pulmonar apresentava uma massa oval de mesma coloração e consistência, com superfície lisa, medindo 5,2 x 2,9 cm (Figura 2B).

As culturas microbiológicas das duas massas intratorácicas não resultaram em crescimento de nenhum microrganismo. O exame histopatológicoindicou áreas de diferenciação condroide, focos osteoides e morfologia celular sugestivos de osteossarcoma condroblástico em região mediastínica, com invasão e envolvimento de diafragma e parênquima pulmonar (Figura 2C). O fragmento ósseo analisado apresentou amplos focos de compactação tecidual, hemorragia peritrabecular e mineralização de tecido osteoide, permeado por plasmócitos e linfócitos típicos. 


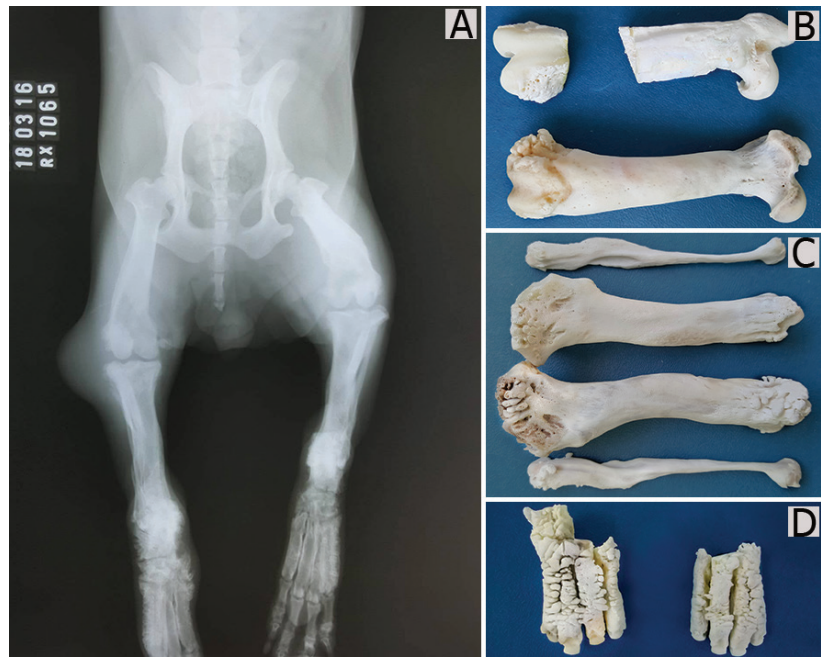

Figura 1. Comparação entre achados radiográficos e necropsiais (peças maceradas e clareadas). A- Radiografia ventrodorsal de pelve e membros posteriores. Nota-se reações periostais no ílio direito e bilaterais em fêmur, tíbia, ossos do tarso, metatarso e falanges proximais. B- Neoformação óssea em couve-flor, bilateral nas epífises proximais e diáfise de fêmur. C- Epífises proximais e distais de tíbia e fíbula ao longo dos metatarsos (D).

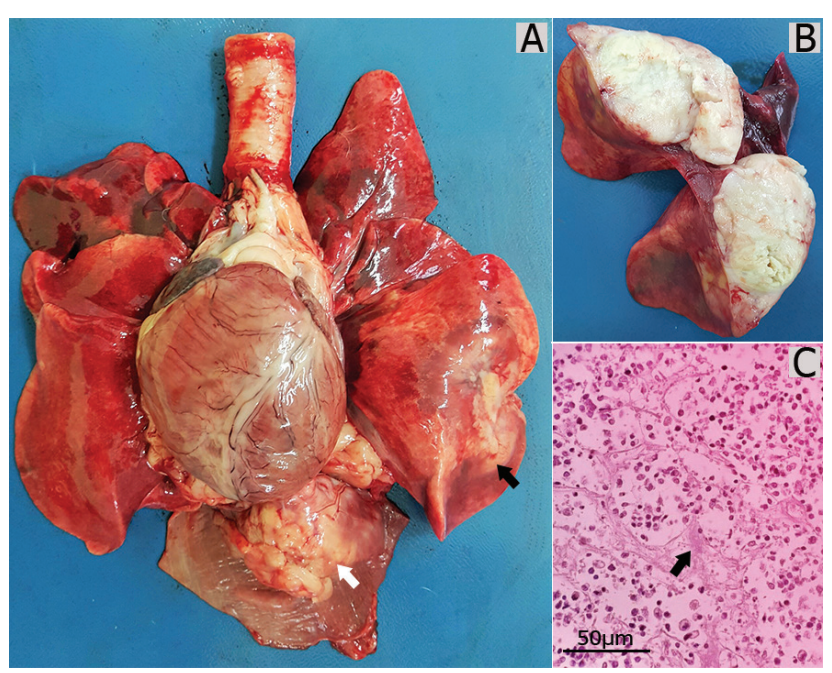

Figura 2. Osteossaroma extraesquelético intratorácico. A- Visibilização do tumor mediastínico (seta branca), junto ao diafragma, e metástase pulmonar (seta preta). B-Corte transversal da massa pulmonar, evidenciando consistência sólida. C- Imagem do exame histopatológico de fragmento da massa mediastínica, evidenciando a matriz osteoide formada (seta). [H\&E, 100x].

\section{DISCUSSÃO}

Duas doenças incomuns ocorreram neste caso: osteossarcoma condroblástico extraesquelético mediastínico e $\mathrm{OH}$ com invasão articular e avanço proximal do esqueleto apendicular. Embora a $\mathrm{OH}$ seja por vezes descrita como uma doença que acomete a diáfise dos ossos longos distais dos membros $[2,9,16]$, este relato descreve também o acometimento de epífises ósseas e ossos proximais dos membros, incluindo o ílio. Assim, a $\mathrm{OH}$ pode progredir proximalmente pelos membros até a pelve [1,3] e ocasionalmente ocorrer em vértebras e no crânio [3]. Além disso, a $\mathrm{OH}$ pode acometer as articulações na medida em que as anormalidades ósseas avançam para as epífises, de modo que o termo osteartropatia hipertrófica não deve ser uma definição tida como errônea na medicina veterinária e exclusiva da medicina humana, como sugerido por alguns autores $[4,16]$. A patogenia da $\mathrm{OH}$ carece de mais estudos para seu total entendimento. As teorias aceitas até o momento envolvem mecanismos neurogênicos, humorais e relacionados a fatores de crescimento [3]. O amplo espectro de doenças de base e a raridade da síndrome são importantes desafios à pesquisa.

Massas mediastínicas, como encontradas neste caso, são reportadas como importante causa de $\mathrm{OH}$ [12]. No entanto, casos de osteossarcomas mediastínicos são extremamente raros. Até onde os autores têm conhecimento, este é o primeiro relato de caso de $\mathrm{OH}$ secundária a osteossarcoma condroblástico extraesquelético mediastínico. A ocorrência de osteossarcoma mediastínico em cães, é descrita em pericárdio [13], coração [11,15], linfonodo [6] e relacionada com a transformação neoplásica de nódulo esofágico de Spirocerca lupi [10].

A maioria dos osteossarcomas extraesqueléticos em cães ocorre em órgãos viscerais, localização diferente da observada neste caso, em que o acometimento foi diafragmático. Osteossarcomas de tecidos moles são mais comumente observado em humanos[18]. A aparência histológica dos osteossarco mas pode variar muito $[14,18]$. O diagnóstico definitivo é dado com base na detecção da produção de matriz osteoide ou óssea pelas células neoplásicas e, no caso de osteossarcomas condroblásticos, pela produção adicional de matriz condroide [14], como observado no exame histológico do paciente relatado. Embora uma minoria de cães com lesões intratorácicas desenvolva $\mathrm{OH}$, cães com metástases pulmonares por osteossarcoma são mais suscetíveis a desenvolver a síndrome do que aqueles com carcinomas metastáticos [3].

$\mathrm{O}$ tratamento para $\mathrm{OH}$ é possível, devendo eliminar a doença de base $[3,12]$. A vagotomia ipsilateral à lesão, ou no lado mais acometido, bem como ressecções cirúrgicas de lesões pulmonares podem rapidamente aliviar a dor, o inchaço de tecidos moles e induzir a regressão das anormalidades ósseas ao longo do tempo [1,9]. No entanto, a terapia para osteossarcomas extraesqueléticos, baseada na remoção cirúrgica possui prognóstico ruim (90 dias), e sua associação com tratamento quimioterápico necessita de mais estudos [5]. 
Os sinais clínicos consequentes a $\mathrm{OH}$ trouxeram o paciente ao serviço veterinário, entretanto, a deterioração clínica pelo avançado osteossarcoma inviabilizou a tentativa terapêutica. O diagnóstico precoce da $\mathrm{OH}$ pode antecipar a detecção e favorecer a intervenção nas diferentes doenças de base relacionadas à síndrome, como neoplasias e abscessos. Em vista disso, conclui-se que para o diagnóstico de $\mathrm{OH}$, deve-se atentar não somente ao exame da porção distal dos membros, visto que o acometimento articular, apendicular proximal e axial também é possível. Conclui-se ainda que, embora raros, osteossarcomas extraesqueléticos devem ser considerados no diagnóstico diferencial de massas intratorácicas em cães com $\mathrm{OH}$.

\section{MANUFACTURERS}

${ }^{1}$ Agener União Saúde Animal. São Paulo, SP, Brazil.

${ }^{2}$ Laboratório Bio-Vet S/A. Vargem Grande Paulista, SP, Brazil.

${ }^{3}$ MSD Saúde Animal. Cruzeiro, SP, Brazil.

Declaration of interest. The authors report no conflicts of interest. The authors alone are responsible for the content and writing of the paper.

\section{REFERENCES}

1 Allan G. 2013. Radiographic signs of joint disease in dogs and cats. In: Thrall D.E. (Ed). Textbook of Veterinary Diagnostic Radiology. 6th edn. Saint Louis: Saunders, pp.341-348.

2 Cetinkaya M.A., Yardimci B. \& Yardimci C. 2001. Hypertrophic osteopathy in a dog associated with intra-thoracic lesions: a case report and a review. Veterinarni Medicina. 56(12): 595-601.

3 Craig L.E, Dittmer K.E. \& Thompson K.G. 2016. Bones and Joints. In: Maxie M.G. (Ed). Jubb, Kennedy, and Palmer's Pathology of Domestic Animals. 6th edn. Saint Louis: Elsevier, pp.17-163.

4 DeCamp C.E., Johnston S.A, Déjardin L.M \& Schaefer S.L. 2016. Disease conditions in small animals. In: DeCamp C.E., Johnston S.A, Déjardin L.M \& Schaefer S.L. (Eds). Brinker, Piermattei and Flo's Handbook of Small Animal Orthopedics and Fracture Repair. 5th edn. Saint Louis: Saunders, pp.821-838

5 Duffy D., Selmic L.E., Kendall A.R \& Powers B.E. 2015. Outcome following treatment of soft tissue and visceral extraskeletal osteosarcoma in 33 dogs: 2008-2013. Veterinary Comparative Oncology. 15(1): 46-54.

6 Hellmen E. 2014. Canine Mammary Osteosarcomas. Journal of Veterinary Science and Technology. 5(2): 163-169.

7 Johnson K.A. 2009. Skeletal diseases. In: Ettinger S.J. \& Feldman E.C. (Eds). Textbook of Veterinary Internal Medicine. 7th edn. Saint Louis: Saunders, pp.819-846.

8 Leonardi L., Roperto F., Salda L.D. \& Mandara M.T. 2012. An uncommon case of extraskeletal canine osteosarcoma. Journal of Comparative Pathology. 146(1): 88-88.

9 Mahesh V. \& Raidurg R. 2014. Hypertrophic osteopathy in a dog secondary to intra-thoracic lesion: a case report. Indian Journal of Canine Practice. 6(2): 156-157.

10 Pazzi P., Tompkins S. \& Kirberger R.M. 2013. Canine spirocercosis-associated extraskeletal osteosarcoma with central nervous system metastasis. Journal of the South African Veterinary Association. 84(1): 1-4.

11 Ramoo S. 2013. Hypertrophic osteopathy associated with two pulmonary tumours and myocardial metastases in a dog: a case report. New Zealand Veterinary Journal. 61(1): 45-48.

12 Salyusarenko M., Peeri D., Bibring U., Ranen E., Bdolah-Abram T. \& Aroch I. 2013. Hypertrophic osteopathy: a retrospective case control study of 30 dogs. Israel Journal of Veterinary Medicine. 68(4): 209-217.

13 Sato T., Koie H., Shibuya H. \& Suzuki K. 2004.Extraskeletal osteosarcoma in the pericardium of a dog. Veterinary Record. 155(24): 780-781.

14 Thompson K.G. \& Pool R.R. 2008. Tumor of bones. In: Meuten D.J. (Ed). Tumors in domestic animals. 4th edn. Ames: Iowa State Press, pp.245-317.

15 Timian J., Yoshimoto S.K. \& Bruyette D.S. 2011. Extraskeletal osteosarcoma of the heart presenting as infective endocarditis. Journal of the American Animal Hospital Association. 47(2): 129-132.

16 Trost M.E, Kommers G.D., Silva T.M, Irigoyen L.F., Fighera R.A., Barros C.S.L. \& Inkelmann M.A. 2012. Osteopatia Hipertrófica em sete cães. Pesquisa Veterinária Brasileira. 32(5): 424-429.

17 Trout N.J. 2015. Hypertrophic osteodystrophy. In: Cote E. (Ed.). Clinical veterinary advisor: dogs and cats. 3rd edn. Saint Louis: Mosby, pp.521-522.

18 Woldemeskel M.W. \& Grice C.D. 2011. Pathology in practice: extraskeletal chondroblastic osteosarcoma. Journal of American Veterinary Medical Association. 238(3): 297-299. 